\title{
Long-term Monitoring of the Changes in Signal-Averaged ECG after Coronary Artery Occlusion and Intracoronary Endothelin-1 Injection in Dogs
}

\author{
P. HUBKA, M. BERNADIČ, I. HULÍN \\ Institute of Pathophysiology, Faculty of Medicine, Comenius University, Bratislava, \\ Slovak Republic
}

Received December 17, 2004

Accepted April 20, 2005

On-line available April 26, 2005

\begin{abstract}
Summary
Myocardium undergoes functional changes in the infarcted region primarily due to ischemia. Following myocyte functional alterations of the noninfarcted myocardium are caused by remodelling and hypertrophy. We have monitored and compared changes in the electrocardiographical $(E C G)$ image after coronary artery occlusion $(\mathrm{CAO}, \mathrm{n}=5)$ and intracoronary endothelin-1 (ET-1, $\mathrm{n}=3$ ) administration during a 6-month period. In 3 dogs, the CAO was repeated 6 months after the first occlusion. Signal-averaged ECG (SA ECG) was recorded before the operation and 10 days, 1 month, 3 months and 6 months after myocardial infarction (MI). The modified Wigner distribution was used for spectrotemporal analysis of the SA ECG. Eight-hour Holter monitoring was performed in each dog before and after experimental MI. Spectrotemporal representations of the QRS complex were stabilized after the first 1-month period in the group of dogs after CAO. The same results were also observed after the repeated CAO. No arrhythmias were recorded 9 days after CAO. The spectrotemporal representations of the QRS complex after intracoronary ET-1 administration were not stabilized during the whole observed period. Very few arrhythmic events were recorded by Holter monitoring already 3 days after intracoronary ET-1 injection. Experimental MI induced by CAO caused a changed ECG image, which was stable from 1 month after MI induction till the end of the monitoring. However, the ECG image after ET-1 administration was not stable during the whole observed period. No arrhythmic events were recorded in either group 3 months postoperatively that could be caused by healthy myocardial status before the experimental MI induction. In clinical practice, however, ischemic heart disease usually precedes the MI. Arrhythmogenic substrate could thus be a consequence of combination of healthy status of the myocardium before MI and MI itself.
\end{abstract}

Key words

SA ECG $\bullet$ Myocardial ischemia $\bullet$ Myocardial infraction $\bullet$ Time-frequency domain $\bullet$ Endothelin-1 $\bullet$ Spectro-temporal analysis 


\section{Introduction}

Acute myocardial ischemia affects the properties of membrane ionic currents and cell-to-cell coupling. Ionic current changes may alter electrical cellular properties of the whole region affected by ischemia (Shaw and Rudy 1997, Priori et al. 1999). Reduced membrane excitability, prolonged action potential duration and slowed conduction (reduced conduction velocity) could affect the cardiac electrical field and its image in the early phase after myocardial infarction (MI). The surrounding non-infarcted myocardium undergoes significant structural changes following MI (Qin et al. 1996). These changes evoke alterations in the electrical properties of myocytes, which could play a key role in arrhythmia development. The electrical field of the postMI heart could be modified due to late changes of the non-infarcted remodeled myocardium.

The signal-averaged ECG and especially late potentials (LP) have been considered a noninvasive marker of delayed and/or fractionated action potential propagation in the myocardium (Buckingham et al. 1989, Cain et al. 1996, Lander et al. 1990). However, the low predictive value of LP (low specificity and sensitivity) has lead to studies dealing with more accurate signal processing techniques (parametric methods, nonstationary methods) in order to enhance the stratification of patients prone to fatal arrhythmias (Cain et al. 1991, Dickhaus et al. 1994, Hulín et al. 1993, Jones et al. 1992, Kavesh et al. 2000, Slavkovský and Hulín 1994, Vazquez et al. 1998, Bernadič et al. 2005). Analysis of the entire QRS complex (not only its terminal part reflected as LP) seemed to be a good approach.

Coronary artery occlusion (CAO) has been used as a standard model for MI. This model is used for induction of functional and morphological changes of myocardium that involve not only the necrotizing infarcted area but the whole myocardium as a consequence of myocardial remodeling. The influence of endothelin-1 (ET-1) on myocardium was recently studied (Becker et al. 2000). The arrhythmogenic effect of plasma ET-1 level on myocardium could be, however, caused not only by its vasoconstrictive effect on coronary vessels but also by its possible direct electrophysiologic effect on myocytes (Miyauchi and Masaki 1999). In the present paper, we described overall changes in the electrocardiological image of the cardiac electrical field during the 6 months follow-up in dogs after CAO and intracoronary ET-1 injection.

\section{Methods}

\section{Experimental myocardial infarction}

Mongrel dogs ( $\mathrm{n}=9$, body weight $12-15 \mathrm{~kg}$ ) were anesthetized with pentobarbital i.v. administration $(25 \mathrm{mg} / \mathrm{kg})$ before intubation and ventilation. Left lateral thoracotomy was performed through the fourth intercostal space. Before opening and fixing the pericardium we injected $3 \mathrm{ml}$ of procainamide beneath it. MI was induced by two methods: i) occlusion of the left anterior descending (LAD) coronary artery between the first and second diagonal branches $(n=5)$, and ii) intracoronary endothelin-1 injection (400 pmol) into the branch of the $\operatorname{LAD}(n=3)$.

The pericardium was then closed, air was excluded from the left pleural cavity after chest closure and dogs were extubated. In 3 dogs, CAO was repeated after a 6-month period. In one dog we performed a sham operation (the whole process was performed except CAO or ET-1 injection). Penicillin and streptomycin were administered i.m. in the postoperative period. The ethical norms for experimental studies in animals were followed throughout the experiment. Experimental procedure was approved by the Ethic Commission of the Faculty of Medicine, Comenius University, Bratislava, Slovak Republic. Animals were under supervision of a veterinarian during the whole experiment.

\section{High-resolution electrocardiography}

The SA ECG was recorded under complete anesthesia. Anesthesia was induced by pentobarbital administration (Pentobarbital Spofa) in a dose of $25 \mathrm{mg} / \mathrm{kg}$ body weight. During the recording, dogs were lying on their right side. Subcutaneous needle electrodes were used. The horizontal lead (X) was placed in the 4th intercostal space on the left and right side. The vertical lead (Y) was placed on the proximal part of the sternum and above the os sacrum. The sagital lead ( $Z$ ) was placed in the 4th intercostal space parasternaly and on the corresponding position paravertebraly on the left.

The Arrhythmia Research Technology model 1200 EPX device was used to record simultaneous orthogonal $\mathrm{X}, \mathrm{Y}$ and $\mathrm{Z}$ leads. The signal from each lead was A-D converted at 12-bit accuracy with sampling rate of $1000 \mathrm{~Hz}$. Ectopic or noisy beats were rejected. At least 100 beats were averaged with mean noise level less than $1.0 \mu \mathrm{V}$. After averaging, the data were stored and transferred to a PC computer. The SA ECG was recorded 10 days, 1 month, 3 months and 6 months after the MI 
induction, respectively. In 3 dogs, SA ECG was also recorded 10 days, 1 month, 3 months, and 6 months after the second CAO, respectively.

\section{Spectrotemporal mapping}

The Wigner distribution (WD) was used in order to obtain the spectrotemporal representation of the SA ECG signal (Martin and Flandrin 1985, Cohen 1989, Novak et al. 1994). Spectrotemporal maps (STM) give us an opportunity to have a complex overview of the contribution of particular frequencies during cardiac activation. The resolution of STM as a result of WD is more precise in both domains (time and frequency) in comparison with STM resulting from a short time FFT (spectrograms). Time and frequency resolution of the short time FFT depends on the used window function. WD offers an algorithm for computing the frequency spectrum without the need of any window function. Furthermore, FFT suffers from smearing and side-lobe leakage. Cross-terms in WD were partially eliminated by smoothing (Choi-Williams filtering was used in our study) (Choi and Williams 1989).

The STM of the particular recording after MI (STMMI) and STM of the SA ECG signal before MI induction in the same $\operatorname{dog}\left(\mathrm{STM}_{\mathrm{O}}\right.$ - original STM) were normalized and fitted (shifted in time and frequency axes in order to achieve the best correlation between $\mathrm{STM}_{\mathrm{O}}$ and $\mathrm{STM}_{\mathrm{MI}}$ ) and then subtracted. The result is a differential STM, which allows us to track differences between particular STMs recorded from the same animal.

\section{Holter monitoring}

Eight-hour Holter monitoring was recorded in all 9 dogs preoperatively and 3-4 days, 8-9 days and 3-6 months after the first operation respectively in the conscious state. In 3 dogs that had undergone the repeated CAO, Holter monitoring was recorded 3-4 and 8-9 days after the second operation.

\section{Results}

\section{Spectrotemporal mapping}

After CAO, changes in the ventricular activation images were stabilized after the transient early phase period and persisted up to 6 months during follow up. The STM image of the ventricular activation was either changed or returned back to its pre-MI image. In both cases, however, the STM image remained unchanged from 1 month after coronary artery occlusion till the end of the follow-up (Fig. 1A). Similar changes were observed in sham-operated animals. The STM image returned back to the preoperative one and remained stable afterwards. No significant changes occurred after the second CAO in 3 dogs and the STM image was stabilized during the whole observed period.
A.

10 days after $\mathrm{CAO}$

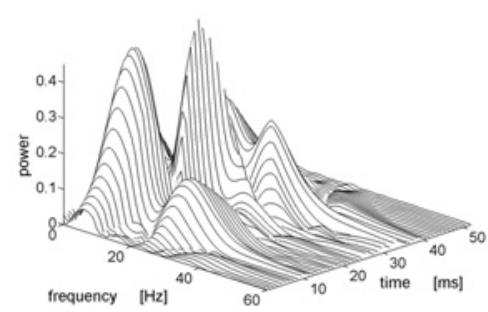

B.

10 days after i.c. ET-1 injection

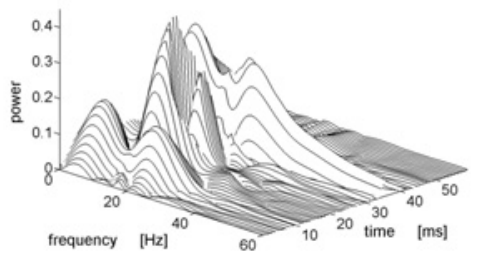

3 months after $\mathrm{CAO}$

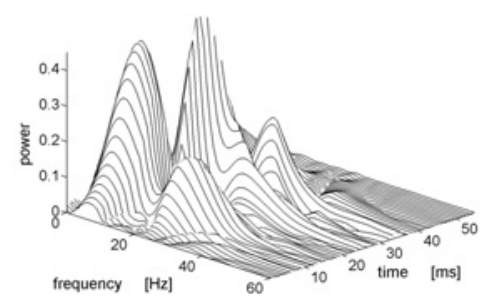

3 months after i.c. ET-1 injection

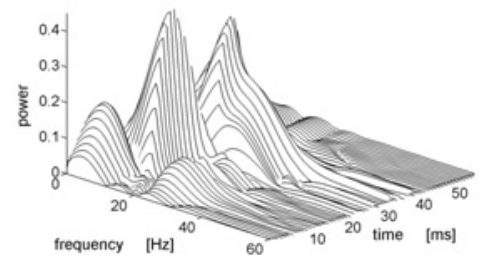

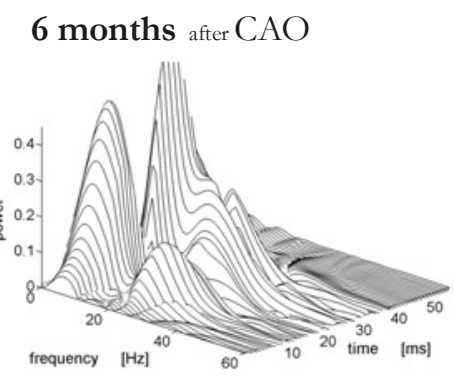

6 months after i.c. ET-1 injection

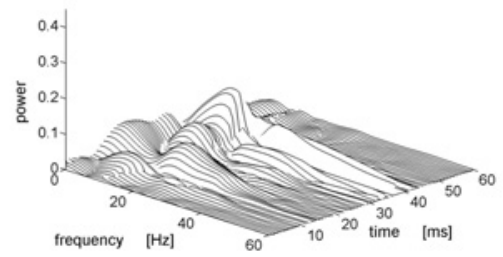

Fig. 1. STM images of the dog 10 days, 3 months and 6 months after MI induction differentiated to pre-MI STM of the same dog. A differential STM images of the dog after CAO, B - differential STM images of the dog after intracoronary endothelin-1 injection. 
Table 1. Number of ventricular extrasystoles observed during Holter monitoring.

\begin{tabular}{|c|c|c|c|c|c|c|}
\hline \multirow{3}{*}{ Experimental Model } & \multirow{3}{*}{ Pre MI } & \multicolumn{5}{|c|}{ Number of ventricular extrasystoles } \\
\hline & & \multicolumn{3}{|c|}{ After 1st MI } & \multicolumn{2}{|c|}{ After 2nd MI } \\
\hline & & 3-4 days & 8-9 days & 3-6 months & 3-4 days & 8-9 days \\
\hline$C A O$ & 0 & 0 & 0 & 0 & 0 & 0 \\
\hline$C A O$ & 0 & 4789 & 320 & 0 & 5901 & 364 \\
\hline$C A O$ & 0 & 2141 & 0 & 0 & 11321 & 3 \\
\hline$C A O$ & 0 & 547 & 0 & 0 & NA & NA \\
\hline$C A O$ & 0 & 456 & 0 & 0 & NA & NA \\
\hline ET-1 & 0 & 0 & 0 & 0 & NA & NA \\
\hline ET-1 & 0 & 1 & 0 & 0 & NA & NA \\
\hline ET-1 & 0 & NA & 4 & 0 & NA & NA \\
\hline Sham & 0 & 0 & 0 & 0 & NA & NA \\
\hline
\end{tabular}

NA - data are not available

The changes between the ECG images after intracoronary ET-1 administration in comparison to the preoperative recording were not stable during the whole observation period, as was observed in the CAO group (Fig. 1B). No transient early phase period, as described in the CAO group, was observed in these animals.

\section{Holter monitoring}

In the Holter monitoring, ventricular extrasystoles were present 3 days after CAO in 4 dogs (Table 1). In the sham-operated dog and one MI dog no extrasystoles were present 3 days after the operation. Significantly smaller numbers of extrasystoles occurred 8 days after CAO. Few arrhythmia events were observed after i.c. ET-1 injection (Table 1). No ectopy was observed with Holter monitoring before $\mathrm{CAO}$ in all dogs.

\section{Discussion}

Studies dealing with cellular level changes (alterations in membrane ionic currents) have recently been published (Aimond et al. 1999, Pinto and Boyden 1999). Developmental alteration in membrane ionic channels and/or in cellular coupling through gap junctions is present in the early phases after coronary artery occlusion. Cellular uncoupling (caused by gap junctions) and reduction of membrane excitability occur in the infarcted region and cause delayed ventricular conduction (Shaw and Rudy 1997). If good cellular coupling through the gap junction is present (sodium current plays a primary role in AP conduction) intrinsic heterogeneity of myocardium is not important from the conduction block point of view (Priori et al. 1999). On the other hand, heterogeneity of the myocardium (no matter whether it was present before $\mathrm{MI}$ or as a consequence of MI) could be the cause of conduction block and consecutive arrhythmia when delayed conduction (e.g. due to cellular uncoupling after MI) is present.

Two pathophysiological conditions should be considered for arrhythmic risk stratification of the patient after a MI: 1) delayed and/or fractionated conduction (possibly detectable by SA ECG and spectrotemporal mapping and using advanced signal processing techniques), and 2) myocardial heterogeneity. This phenomenon could possibly be detected by monitoring of beat-to-beat alternations (alternations in spectrotemporal images of the QRS complex and/or analysis of $\mathrm{T}$ wave and/or T wave alternations) (Smith et al. 1988, Verrier and Nearing 1994, Atiga et al. 1998, Chinushi et al. 1998).

Our results describe the long-term changes in the electrical image of ventricular activation after coronary artery occlusion and intracoronary ET-1 injection. Stabilization of these changes after a certain period after MI suggests that the myocardium itself (myocytes together with gap junctions) is also stabilized. On the other hand, instability in the ECG image in the same subject could mirror the instability in the myocardium, which could be considered as a risk factor for arrhythmia development. From this point of view, comparison of consecutive SA ECG recordings (and especially their 
spectrotemporal representations) in the same subject could be useful as an indicator of the remodeling state and possibly arrhythmia-prone substrate.

Holter monitoring revealed that there were many arrhythmic events in the group of dogs with MI induced by CAO during the early phase. These findings were accompanied by pronounced changes in the STM image of ventricular activation in comparison to pre-MI STMs. After this early period, however, the changes in the ventricular activation STMs were stabilized and no arrhythmic events were present in the Holter monitoring.

On the other hand, Holter monitoring revealed a very low number of arrhythmic events in dogs after i.c. ET-1 administration during the whole period of follow up. Surprisingly, STM images of ventricular activation were not stabilized during the whole observed period. ET-1 directly affects electrical properties of the myocytes predominantly via paracrine/autocrine mechanism (Miyauchi and Masaki 1999). On the other hand, higher plasma ET-1 levels probably have indirect effects on the electrical milieu of the myocardium via an overall vasoconstriction effect on the cardiac vessels (Kurihara et al. 1989, Larkin et al. 1989, Šídlo et al. 1996). Higher plasma ET-1 levels could, however, induce changes in other organs (e.g. lung and renal functions) which could in turn affect cardiac function and cause a changing ventricular activation image, which could not be arrhythmogenic (Miyauchi and Masaki 1999). Furthermore, higher plasma ET-1 levels could have potentially different effects on healthy myocardium than on the ischemic myocardium.

Acute MI in men occurs after a previous period of changed myocardial perfusion that might lead to altered electrical properties. This pre-MI state of myocardium could play an important role in the post-MI period. On the other hand, MI is closely connected with remodeling and with post-MI hypertrophy. The pre-MI myocardium state, evolution of the infarcted region together with remodeling of the non-infarcted area could play a deterministic role from the post-MI arrhythmia point of view. Analysis of the electrical changes after MI could provide useful additional information (along with other diagnostic methods), which could be helpful in the risk stratification of $\mathrm{MI}$ patients prone to malignant arrhythmias.

\section{Acknowledgements}

This study was supported by grant no. 1/4114/97 and $1 / 7537 / 20$ from Ministry of Education of the Slovak Republic.

\section{References}

AIMOND F, ALVAREZ JL, RAUZIER JM, LORENTE P, VASSORT G: Ionic basis of ventricular arrhythmias in remodeled rat heart during long-term myocardial infarction. Cardiovasc Res 42: 402-415, 1999.

ATIGA WL, CALKINS H, LAWRENCE JH, TOMASELli GF, SMITH JM, BERGER, RD: Beat-to-beat repolarization lability identifies patients at risk for sudden cardiac death. J Cardiovasc Electrophysiol 9: 899908, 1998.

BECKER R, MERKELY B, BAUER A, GELLER L, FAZEKAS L, FREIGANG KD, VOSS F, SENGES JC, KUEBLER W, SCHOELS W: Ventricular arrhythmias induced by endothelin-1 or by acute ischemia: a comparative analysis using three-dimensional mapping. Cardiovasc Res 45: 310-320, 2000.

BERNADIC M, HUBKA P, SLAVKOVSKY P, PETRASOVA H, HOLZEROVA J, MAASOVA D, ZLATOS ZLATOS L, MLADOSIEVICOVA B, HULIN I: High resolution electrocardiography in healthy dogs: time domain parameters and comparison of the non-stationary (Wigner distribution) versus standard stationary frequency domain analysis methods. Physiol Res 54: 477-484, 2005.

BUCKINGHAM TA, THESSEN CM, HERTWECK D, JANOSIK DL, KENNEDY HL: Signal-averaged electrocardiography in the time and frequency domains. Am J Cardiol 63: 820-825, 1989.

CAIN ME, AMBOS HD, MARKHAM J, LINDSAY BD, ARTHUR RM: Diagnostic implications of spectral and temporal analysis of the entire cardiac cycle in patients with ventricular tachycardia. Circulation 83: 1637$1648,1991$.

CAIN ME, ANDERSON J, ARNSDORF MF, MASON JW, SCHEINMAN MM, WALDO AL: Signal-averaged electrocardiography. J Am Coll Cardiol 27: 238-249, 1996.

CHINUSHI M, RESTIVO M, CAREF EB, EL SHERIF N: Electrophysiological basis of arrhythmogenicity of QT/T alternans in the long-QT syndrome. Circ Res 83: 614-628, 1998. 
CHOI H, WILLIAMS WJ: Improved time-frequency representation of multicomponent signal using exponential kernels. IEEE Trans Acoust Speech Signal Process 37: 862-871, 1989.

COHEN L: Time-frequency distribution - a review. Proc IEEE 77: 941-981, 1989.

DICKHAUS H, KHADRA L, BRACHMANN J: Time-frequency analysis of ventricular late potentials. Methods Inf Med 33: 187-195, 1994.

HULÍN I, SLAVKOVSKÝ P, HATALA R, PETRÁŠOVÁ H, ČÍK V, MURÍN J: Gliding window fast Fourier transform analysis - a new method for discovering the contribution of higher frequencies in signal-averaged ECG. Can J Cardiol 9: 789-796, 1993.

JONES DL, TOUVANNAS JS, LANDER P, ALBERT DE: Advanced time-frequency methods for signal-averaged ECG analysis. J Electrocardiol 25 (Suppl): 188-194, 1992.

KAVESH NG, SARANG SE, GOLD MR: Signal-averaged isoharmonic body surface maps of patients with ischemic cardiomyopathy. J Cardiovasc Electrophysiol 11: 160-167, 2000.

KURIHARA H, YAMAOKI K, NAGAI R, YOSHIZUMI M, TAKAKU F, SATOH H, INUI J, YAZAKI Y: Endothelin: a potent vasoconstrictor associated with coronary vasospasm. Life Sci 44: 1937-1943, 1989.

LANDER P, ALBERT DE, BERBARI EJ: Spectrotemporal analysis of ventricular late potentials. $J$ Electrocardiol 23: 95-108, 1990.

LARKIN SW, CLARKE JG, KEOGH BE, ARAUJO L, RHODES C, DAVIES GJ, TAYLOR KM, MASERI A: Intracoronary endothelin induces myocardial ischemia by small vessel constriction in the dog. Am J Cardiol 64: 956-958, 1989.

MARTIN W, FLANDRIN P: Wigner-Ville spectral analysis of nonstationary processes. IEEE Trans ASSP 33: 1461$1469,1985$.

MIYAUCHI T, MASAKI T: Pathophysiology of endothelin in the cardiovascular system. Annu Rev Physiol 61: 391$415,1999$.

NOVAK P, LI Z, NOVAK V, HATALA R: Time-frequency mapping of the QRS complex in normal subjects and in postmyocardial infarction patients. J Electrocardiol 27: 49-60, 1994.

PINTO JM, BOYDEN PA: Electrical remodeling in ischemia and infarction. Cardiovasc Res 42: 284-297, 1999.

PRIORI SG, BARHANIN J, HAUER RN, HAVERKAMP W, JONGSMA HJ, KLEBER AG, MCKENNA WJ, RODEN DM, RUDY Y, SCHWARTZ K, SCHWARTZ PJ, TOWBIN JA, WILDE A: Genetic and molecular basis of cardiac arrhythmias; impact on clinical management. Study group on molecular basis of arrhythmias of the working group on arrhythmias of the European Society of Cardiology. Eur Heart J 20: 174-195, 1999.

QIN D, ZHANG ZH, CAREF EB, BOUTJDIR M, JAIN P, EL SHERIF N: Cellular and ionic basis of arrhythmias in postinfarction remodeled ventricular myocardium. Circ Res 79: 461-473, 1996.

SHAW RM, RUDY Y: Electrophysiologic effects of acute myocardial ischemia. A mechanistic investigation of action potential conduction and conduction failure. Circ Res 80: 124-138, 1997.

ŠIDLO J, BERNADIČ M, ZLATOŠ L, HOLZEROVÁ J, MAASOVÁ D, HULÍN I: Morphological findings after intracoronary application of endothelin-1 in dog. Bratisl Lek Listy 97: 611-613, 1996.

SLAVKOVSKÝ P, HULÍN I: Gliding-window fast Fourier transform analysis of the entire QRS complex and the distribution of area ratio peaks in healthy subjects and patients with myocardial infarction. Coron Artery Dis 5 : 249-256, 1994.

SMITH JM, CLANCY EA, VALERI CR, RUSKIN JN, COHEN RJ: Electrical alternans and cardiac electrical instability. Circulation 77: 110-121, 1988.

VAZQUEZ R, CAREF EB, TORRES F, REINA M, HUET J, GUERRERO JA, EL SHERIF N: Comparison of the new acceleration spectrum analysis with other time- and frequency-domain analyses of the signal-averaged electrocardiogram. Eur Heart J 19: 628-637, 1998.

VERRIER RL, NEARING BD: Electrophysiological basis for $\mathrm{T}$ wave alternans as an index of vulnerability to ventricular fibrilation. J Cardiovasc Electrophysiol 5: 445-461, 1994.

\section{Reprint requests}

Peter Hubka, Institute of Pathophysiology, Faculty of Medicine, Comenius University, Sasinkova 4, SK-811 08 Bratislava, Slovak Republic. E-mail: peter.hubka@fmed.uniba.sk 